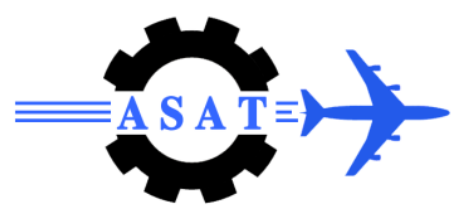

\title{
Performance Analysis of Non-Coherent Multiple-Access Technique Using Different Modulations
}

\author{
A.M.E. Fouda ${ }^{*}$, A. Bassioni ${ }^{\dagger}$, and E.A. Wanees*
}

\begin{abstract}
Direct sequence (DS) and frequency hopping (FH) are two conventional types of spread spectrum multiple access techniques (SSMA). Recently a hybrid combination between them (hybrid SFH/DS SSMA) are attractive because they can combine the advantages while avoiding some of their disadvantages. The paper presents the throughput and delay analysis when it is required to transmit data over a packet switched code division multiple access network based on the hybrid SFH/DS SSMA. The performance is discussed assuming noncoherent reception of synchronous type hybrid system operating through additive white Gaussian noise channels, and employing different modulation techniques, those are binary frequency shift keying BFSK, M-ary FSK with noncoherent demodulation, as well as the system with differential phase shift keying modulation DPSK. Two closed form for the different modulation types are derived. One of them is probability of error (BER) as a function of the signal to noise ratio (SNR), the other one is the system throughput. Moreover, the paper presents a performance comparison between the hybrid SFH/DS SSMA, pure $\mathrm{SFH}$, and pure DS systems taking the throughput and the delay as performance measures.
\end{abstract}

Keywords: DS, FH SSMA, CDMA, throughput, delay

\section{Introduction}

A radio network is a collection of terminals, which can communicate with each other via radio links. A key feature of a radio network is that each radio transmission is a potential source of interference for all terminals within the range of the transmitter. Spread spectrum is a promising signaling scheme in packet radio network due to its various inherent advantages it can combat multi-path fading and provide multiple-access capability. Direct sequence (DS), frequency hopping (FH) and hybrid DS/FH schemes have been proposed for such applications. DS combats efficiently the problem of multi-path fading but suffers from the near-far effect. On the other hand slow frequency hopping (SFH) is more immune to the nearfar effect though more sensitive to multi-path fading. Hybrid systems are attractive because they can combine the advantages of both direct-sequence and frequency hopping systems, while avoiding some of their disadvantages. Thus, hybrid DS/SFH CDMA is a good solution for confronting these problems.

The paper is organized as follows; in section II we introduce to the system model and illustrate its basic principle of operation, describing the basic equation of transmitted and received signals, as well as presenting the BER analysis for the different types of modulations. In section III the performance analysis of the system throughput and delay is

\footnotetext{
* Modern Academy for Engineering\& Technology, Cairo, Egypt, fouda1964@ maktoob.com

† Modern Academy for Engineering \& Technology, Cairo, Egypt.

*Egyptian Armed Forces, Egypt, mohwanees@yahoo.com
} 
evaluated, a closed form expression for the system throughput is derived assuming different modulation types (BFSK, MFSK, and DPSK). In section IV the delay analysis is introduced. While in section $\mathrm{V}$ the numerical results are given and discussed. Finally conclusions are given and discussed throughout section VI.

\section{System Model and BER Analysis}

Assuming asynchronous hybrid SFH/DS SSMA system with noncoherent reception operating through additive white Gaussian noise channels, and employing different modulation techniques (BFSK, M-ary FSK, and DPSK modulations) one at a time.

Figure 1 shows the transmitter model of the hybrid SFH/DS system, including data source generation, the DS spreader, employing one type at a time of the given three modulations (BFSK, MFSK, or DPSK) and the frequency hopper, and finally BPF for noise enhancements.

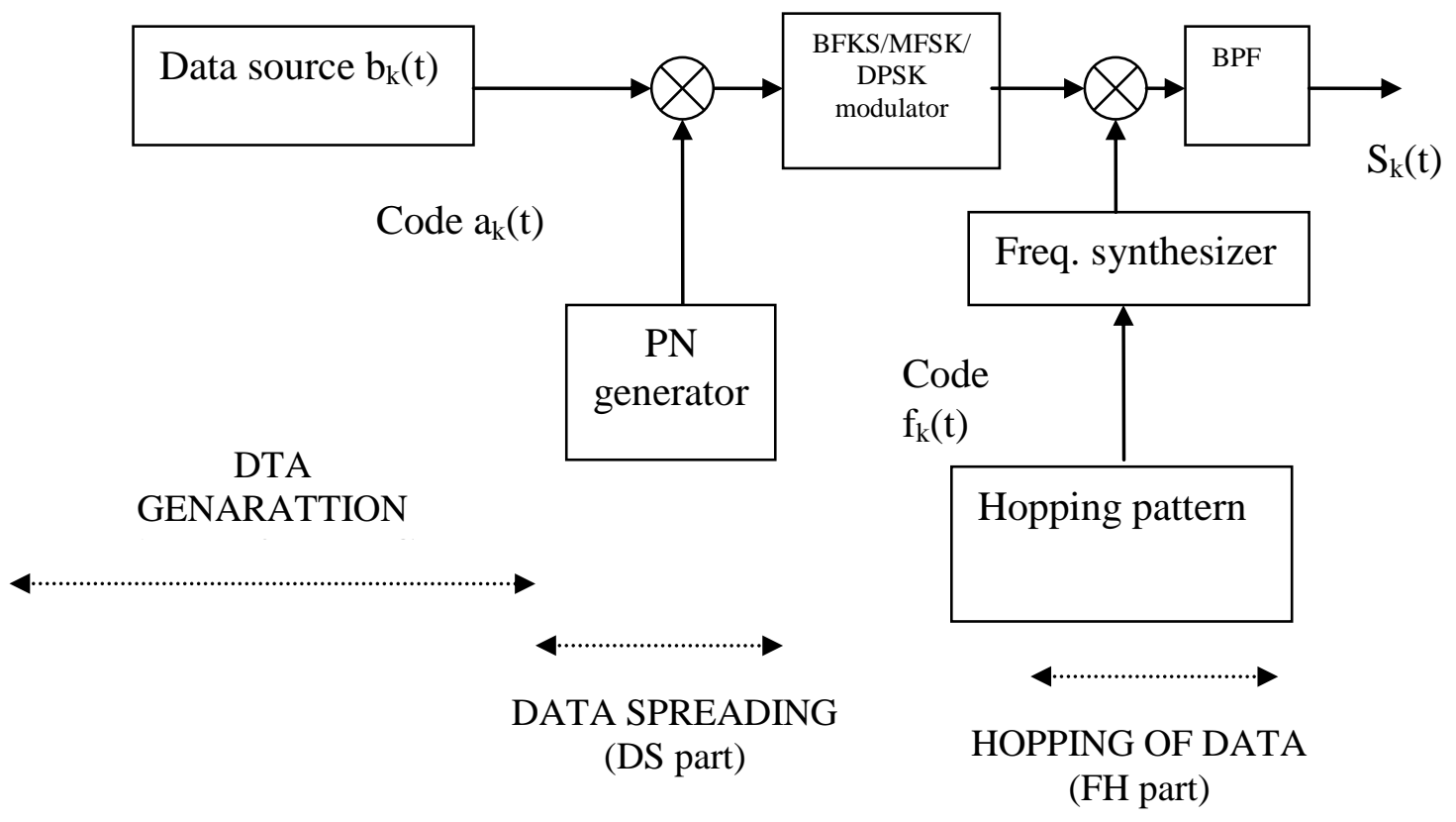

Fig. 1 Transmitter part of the hybrid DS/SFH spread spectrum signal

Assume we have $\mathrm{K}$ number of active users then the transmitted signal of the $\mathrm{K}^{\text {th }}$ user is given as [1]:

$\mathrm{S}_{\mathrm{k}}(\mathrm{t})=\sqrt{2 P} \mathrm{a}_{\mathrm{k}}(\mathrm{t}) \mathrm{b}_{\mathrm{k}}(\mathrm{t}) \cos \left(\omega_{\mathrm{c}} \mathrm{t}+\omega_{\mathrm{k}} \mathrm{t}+\theta_{\mathrm{k}}+\alpha_{\mathrm{k}}(\mathrm{t})\right)$

where $P$ is the average transmitted signal power, and $\omega_{c}=2 \pi f_{c}$ is the carrier frequency common for all users, while $\omega_{\mathrm{k}}(\mathrm{t})$ is the random hopping pattern, it's a first order stationary Markov process, and is derived from a set of q frequencies which is not necessarily equally spaced with minimum spacing $\omega$. Also, $\theta_{\mathrm{k}}$ is the random phase introduced by the $\mathrm{k}^{\text {th }}$ modulator spreader and $\alpha_{k}(t)$ is a random phase introduced by the $\mathrm{k}^{\text {th }}$ user frequency hopper. The term $b_{k}(t)$ is the data bits sequence generated by user $\mathrm{k}$, with the information bit rate $R_{b}=1 / T_{b}$, where $\mathrm{T}_{\mathrm{b}}$ is the information bit duration. The $\mathrm{K}^{\mathrm{th}}$ data signal $b_{k}(t)$ can be expressed as: 
$b_{k}(t)=\sum_{j} b_{k}^{j} P_{T}(t-j T)$

where $b_{k}^{j}$ is the $\mathrm{J}^{\text {th }}$ data bit of the user $\mathrm{K}$, and $b_{k}^{j} \in\{0,1\}$, and $P_{T}(t)$ is the NRZ rectangular pulse of duration $\mathrm{T}$. The spreading sequence term $\mathrm{a}_{\mathrm{k}}(\mathrm{t})$ can be expressed as :

$a_{k}(t)=\sum_{j} a_{k}^{j} P_{T c}\left(t-j T_{c}\right)$

where $a_{k}{ }^{j}$ is the $J^{\text {th }}$ pulse and $a_{k}{ }^{j} \in\{-1,1\}$ and $T_{c}$ is the chip duration of the NRZ rectangular pulse $P_{T c}(t)$. The PN sequence waveform $a_{\mathrm{k}}(\mathrm{t})$ is assumed to be random signature sequence with rate $R_{c}=1 / T_{c}$, and assume $\omega>\left(2 / T_{c}\right)$, so that there is negligible overlapping of the DS signal when hopped to adjacent frequencies. The minimum system bandwidth is $B=2 q / T_{c}=2 N q / T$, where we assume N-Code pulses in each data pulse ( $\left.T=N T_{c}\right)$. After multiplying $b_{k}(t)$ with $a_{k}(t)$, the produced DS spreaded signal is then modulated, and frequency hopped.

As shown from Fig. 2, the receiver model includes the signal corrupted with AWGN noise, so after frequency conversion and filtration the signal will be frequency dehopped, then demodulation process is accomplished and then the DS despreader is introduced. Finally the received output data sequence can be obtained

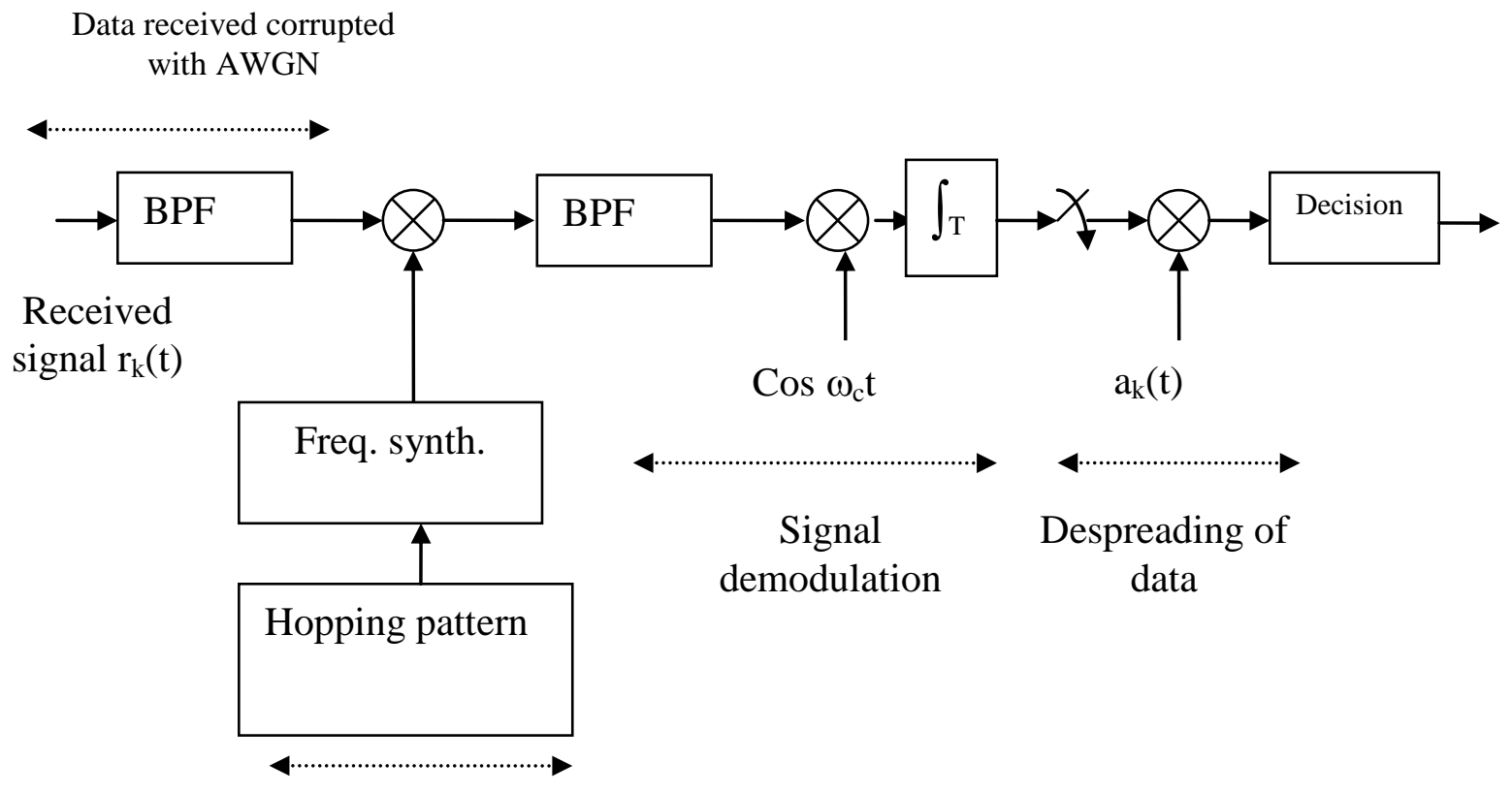

Frequency Dehopping

Fig. 2 Receiver part of the hybrid DS/SFH spread spectrum signal

The form of the received signal for the hybrid system is given as:

$\mathrm{r}_{\mathrm{k}}(\mathrm{t})=\sum \mathrm{Y}_{\mathrm{k}}\left(\mathrm{t}-\tau_{\mathrm{k}}\right)+\mathrm{n}(\mathrm{t})$

Thus the corresponding received signal model from the $\mathrm{k}^{\text {th }} \mathrm{user}$ will be:

$\mathrm{r}_{\mathrm{k}}(\mathrm{t})=\sqrt{2 P} \mathrm{~b}_{\mathrm{k}}\left(\mathrm{t}-\tau_{\mathrm{K}}\right) \mathrm{a}_{\mathrm{k}}\left(\mathrm{t}-\tau_{\mathrm{K}}\right) \cos \left\{2 \pi\left(\mathrm{f}_{\mathrm{c}}+\mathrm{f}_{\mathrm{hk}}\left(\mathrm{t}-\tau_{\mathrm{K}}\right)\right) \mathrm{t}+\theta_{\mathrm{K}}\left(\mathrm{t}-\tau_{\mathrm{K}}\right)+\alpha_{\mathrm{K}}\left(\mathrm{t}-\tau_{\mathrm{K}}\right)\right\}+\mathrm{n}(\mathrm{t})$ 
where $n(t)$ is the AWGN with two sided power spectral density $\mathrm{N}_{\mathrm{o}} / 2, \tau_{\mathrm{k}}$ is the time delay and is uniformly distributed on $\left[0, \mathrm{~T}_{\mathrm{b}}\right]$. The error probability of the hybrid SSMA system can be expressed as [2]:

$\mathrm{P} 1=\sum_{k_{f}=0}^{K-1} \sum_{k_{p}=0}^{K-1-k_{f}} \mathrm{P}_{\mathrm{h}}\left(\mathrm{k}_{\mathrm{f}}, \mathrm{k}_{\mathrm{p}}\right) \mathrm{p}_{\mathrm{e}}\left(\mathrm{k}_{\mathrm{f}}, \mathrm{k}_{\mathrm{p}}\right)$

where $\mathrm{p}_{\mathrm{e}}\left(\mathrm{k}_{\mathrm{f}}, \mathrm{k}_{\mathrm{p}}\right)$ is the conditional error probability of the system, given that $\mathrm{k}_{\mathrm{f}}$ full hits and $\mathrm{k}_{\mathrm{p}}$ partial hits occurred. While $\mathrm{p}_{\mathrm{h}}\left(\mathrm{k}_{\mathrm{f}}, \mathrm{k}_{\mathrm{p}}\right)$ is the probability of the occurrence of $\mathrm{k}_{\mathrm{f}}$ full hits, and $\mathrm{k}_{\mathrm{p}}$ partial hits from the other K-1 users. For independent hopping patterns the joint probability of $\mathrm{X}$ full hits and $\mathrm{Y}$ partial hits is given by:

$P_{h}(X, Y)=\left(\begin{array}{l}K-1 \\ X\end{array}\right)\left(\begin{array}{l}K-1-X \\ Y\end{array}\right) P_{f}^{X} P_{p}^{Y}\left(1-P_{f}-P_{p}\right)^{K-1-X-Y}$

where $0 \leq \mathrm{X} \leq \mathrm{K}, 0 \leq \mathrm{Y} \leq \mathrm{K}-\mathrm{X}$ and $\mathrm{P}_{\mathrm{f}}$ and $\mathrm{P}_{\mathrm{p}}$, denote the probability of full and partial hits from other users, respectively. These probabilities have been calculated in [3] for asynchronous hybrid systems, first-order Markov random hopping patterns, and AWGN channels they are given by:

$\mathrm{P}_{\mathrm{f}}=\left(1-\mathrm{N}_{\mathrm{b}}^{-1}\right) \mathrm{q}^{-1}$

and

$\mathrm{P}_{\mathrm{p}}=2 \mathrm{~N}_{\mathrm{b}}^{-1} \mathrm{q}^{-1}$

where q represents number of hopping frequencies, and $\mathrm{N}_{\mathrm{b}}$ is the number of bits per frequency hop. While in the synchronous case any user k can cause only full hits, , then we get:

$P_{h}=q^{-1}$

Thus for the first-order Markov and memoryless random hopping patterns we can use the following modification form of equation (5) to get the new form as [4] :

$\mathrm{P}_{\mathrm{et}}=\sum_{k=0}^{K-1}\left(\begin{array}{l}K-1 \\ k\end{array}\right) \mathrm{P}_{\mathrm{h}}{ }^{\mathrm{k}}\left(1-\mathrm{P}_{\mathrm{h}}\right)^{\mathrm{K}-1-\mathrm{k}} \mathrm{P}_{\mathrm{e}}$

where $\mathrm{P}_{\mathrm{e}}$ is the conditional probability of error given that $\mathrm{k}$ full hits from other users occur. It has to be calculated for the different modulations under consideration.

For the hybrid system employing BFSK the conditional probability can be expressed as [4]:

$\mathrm{P}_{\mathrm{e}}=0.5 \exp \left\{-0.25\left[\left(2 \mathrm{E}_{\mathrm{b}} / \mathrm{N}_{0}\right)^{-1}+\mathrm{k} / 4 \mathrm{~N}\right]^{-1}\right\}$

Then the unconditional average error probability of synchronous hybrid system employing BFSK:

$P_{e B F S K}=0.5 \sum_{k=0}^{K-1}\left(\begin{array}{l}K-1 \\ k\end{array}\right) \mathrm{q}^{-\mathrm{k}}\left(1-\mathrm{q}^{-1}\right)^{\mathrm{K}-1-\mathrm{k}} \exp \left\{-0.25\left[\left(2 \mathrm{E}_{\mathrm{b}} / \mathrm{N}_{0}\right)^{-1}+\mathrm{k} / 4 \mathrm{~N}\right]^{-1}\right\}$

For the hybrid system employing MFSK the conditional error probability can be expressed as: 


$$
P_{e}=\sum_{k=0}^{K-1}\left(\begin{array}{l}
M-1 \\
k
\end{array}\right) \frac{(-1)^{m+1}}{m+1} \exp \left\{-\frac{m}{2(m+1)}\left[\left(2 E_{b} \log _{2} M / N_{0}\right)^{-1}+\frac{k}{2 M N}\right]^{-1}\right\}
$$

Then the unconditional average error probability of synchronous hybrid system employing MFSK:

$P_{\text {eMFSK }}=\sum_{k=0}^{K-1}\left(\begin{array}{l}K-1 \\ k\end{array}\right) \mathrm{q}^{-\mathrm{k}}\left(1-\mathrm{q}^{-1}\right)^{\mathrm{K}-1-\mathrm{k}} \sum_{k=0}^{K-1}\left(\begin{array}{l}M-1 \\ k\end{array}\right) \frac{(-1)^{m+1}}{m+1} \exp \left\{-\frac{m}{2(m+1)}\left[\left(2 E_{b} \log _{2} M / N_{0}\right)^{-1}+\frac{k}{2 M N}\right]^{-1}\right\}$

For the hybrid system employing DPSK the conditional error probability can be expressed as [5]:

$\mathrm{P}_{\mathrm{e}}=0.5 \exp \left\{-0.5\left[\left(2 \mathrm{E}_{\mathrm{b}} / \mathrm{N}_{0}\right)^{-1}+\mathrm{k} / 2 \mathrm{~N}\right]^{-1}\right\}$

Then the unconditional average error probability of synchronous hybrid system employing DPSK:

$P_{e D P S K}=0.5 \sum_{k=0}^{K-1}\left(\begin{array}{l}K-1 \\ k\end{array}\right) \mathrm{q}^{-\mathrm{k}}\left(1-\mathrm{q}^{-1}\right)^{\mathrm{K}-1-\mathrm{k}} \exp \left\{-0.5\left[\left(2 \mathrm{E}_{\mathrm{b}} / \mathrm{N}_{0}\right)^{-1}+\mathrm{k} / 2 \mathrm{~N}\right]^{-1}\right\}$

Figure 3 shows the different plots for the BER as a function of signal to noise ratio to provide a comparison between the different modulations.

\section{Throughput Performance Analysis of Hybrid System}

For transmission of computer data, a packet communications schedule can be more efficient than using circuit switched protocol (suitable in speech oriented systems). In a packet network, throughput and delay are appropriate parameters, rather than maximum user capacity. The throughput determines the average number of successfully received packets per time slot, given a certain amount of traffic. For a certain amount of throughput it is important to know what will be the average delay of a packet.

Generally, error will occurs and packets are lost if the number of simultaneous active users $(\mathrm{k})$ exceeds the threshold capacity $(\mathrm{C})$. The lost packets are re-scheduled and retransmitted after a sufficient time delay.

The throughput can be calculated as the average number of successfully received packets in the system and can be expressed as the following equation [1]:

$\mathrm{S}=(1 / \mathrm{C}) \sum_{k=1}^{c} \mathrm{k} \mathrm{P}_{\mathrm{r}}(\mathrm{k}) \mathrm{P}_{\mathrm{sk}}$

where

$\mathrm{C}$ is the threshold system capacity and $\mathrm{P}_{\mathrm{k}}$ is the probability that $\mathrm{k}$ packets are transmitted simultaneously. It depends on the traffic model (finite or infinite population model) Assuming the infinite population model, this population is modeled by the Poisson distribution with parameter $G$, where $G$ is the average population size. Since the average 
number of transmitted new and backlogged packets is given by $G=\lambda T$, Then the probability that $\mathrm{K}$ packets are transmitted of the Poisson distribution is given as [6]:

$P_{r}(K)=(K !)^{-1} \cdot G^{K} \cdot \exp (-G)$

$\mathrm{P}_{\mathrm{sk}}$ is the packet success probability if $\mathrm{k}$ packets are transmitted simultaneously. The probability of correctly receiving a packet of $\mathrm{N}_{\mathrm{p}}$ data bits $\mathrm{P}_{\mathrm{sk}}$ is given by [7]:

$\mathrm{P}_{\mathrm{sk}}=\left[1-\mathrm{P}_{\mathrm{er}}(\mathrm{k})\right]^{\mathrm{Np}}$

where $\mathrm{P}_{\mathrm{er}}=\mathrm{P}_{\mathrm{eBFSK}}, \mathrm{P}_{\mathrm{er}}=\mathrm{P}_{\mathrm{eMFSK}}$, and $\mathrm{P}_{\mathrm{er}}=\mathrm{P}_{\mathrm{eDPSK}}$ for the different modulation BFSK, MFSK, and DPSK respectively. Then the closed form expression of the system throughput for the BFSK hybrid SSMA is given in equation (20):

$$
\begin{gathered}
\mathrm{S} 1=(1 / \mathrm{C}) \sum_{k=1}^{c}\left\{\mathrm { k } ( \mathrm { K } ! ) ^ { - 1 } \cdot \mathrm { G } ^ { \mathrm { K } } \cdot \operatorname { e x p } ( - \mathrm { G } ) \left[1-0.5 \sum_{k=0}^{K-1}\left(\begin{array}{l}
K-1 \\
k
\end{array}\right) \mathrm{q}^{-\mathrm{k}}\left(1-\mathrm{q}^{-1}\right)^{\mathrm{K}-1-\mathrm{k}}\right.\right. \\
\left.\left.* \exp \left\{-0.25\left[\left(2 \mathrm{E}_{\mathrm{b}} / \mathrm{N}_{0}\right)^{-1}+\mathrm{k} / 4 \mathrm{~N}\right]^{-1}\right\}\right]^{\mathrm{Np}}\right\}
\end{gathered}
$$

Then the closed form expression of the system throughput for the MFSK hybrid SSMA is:

$$
\begin{aligned}
\mathrm{S} 2=1 / \mathrm{C} & \sum_{k=1}^{c} \mathrm{k}(\mathrm{K} !)^{-1} \cdot \mathrm{G}^{\mathrm{K}} \cdot \exp (-\mathrm{G})\left[1-\sum_{k=0}^{K-1}\left(\begin{array}{l}
K-1 \\
k
\end{array}\right) \mathrm{q}^{-\mathrm{k}}\left(1-\mathrm{q}^{-1}\right)^{\mathrm{K}-1-\mathrm{k}}\right. \\
& \left.* \sum_{k=0}^{K-1}\left(\begin{array}{l}
M-1 \\
k
\end{array}\right) \frac{(-1)^{m+1}}{m+1} \exp \left\{-\frac{m}{2(m+1)}\left[\left(2 E_{b} \log _{2} M / N_{0}\right)^{-1}+\frac{k}{2 M N}\right]^{-1}\right\}\right]^{\mathrm{N}}
\end{aligned}
$$

The closed form expression of the system throughput for the DPSK hybrid SSMA is:

$$
\mathrm{S} 3=1 / \mathrm{C} \sum_{k=1}^{c} \mathrm{k}(\mathrm{K} !)^{-1} \cdot \mathrm{G}^{\mathrm{K}} \cdot \exp (-\mathrm{G})\left[1-0.5 \sum_{k=0}^{K-1}\left(\begin{array}{l}
K-1 \\
k
\end{array}\right) \mathrm{q}^{-\mathrm{k}}\left(1-\mathrm{q}^{-1}\right)^{\mathrm{K}-1-\mathrm{k}} \exp \left\{-0.5\left[\left(2 \mathrm{E}_{\mathrm{b}} / \mathrm{N}_{0}\right)^{-1}+\mathrm{k} / 2 \mathrm{~N}\right]^{-1}\right\}\right.
$$

Figure 5 shows the different plots for the system throughput as a function of offered traffic for these different modulation techniques derived above.

\section{Delay Analysis of a Hybrid SFH/DS SS}

The average delay is defined as the average number of slot times it takes for a packet to be successfully received. Thus it is the average time duration between the packet being offered to the transmitter and the packet being successfully received. The average delay in an indoor network, assuming negligible round trip propagation delay and immediate acknowledgement, can be obtained as [8]:

$\mathrm{D}=1.5+[\mathrm{G} / \mathrm{CS}-1]\left(\left\lfloor\delta_{\tau}+1\right\rfloor+1\right)$

where

- $\mathrm{G} / \mathrm{CS}-1$ is the average number of retransmission for a packet to be successfully received

- $\left\lfloor\delta_{\tau}+1\right\rfloor$ the delay due to each transmission 
- $\delta_{\tau}$ is the mean of the retransmission delay, which is uniformly distributed over the range from which the retransmission delay is selected

$\mathrm{C}$ is the user's threshold capacity

Here it is assumed that the minimum delay for a slotted system (that use slot time protocol for transmission) is 1.5 slot durations. This time consists of one time slot being the average time between the time the packet is offered to the transmitter and the beginning of the next slot.

Figure 6 shows the delay in of the considered hybrid DS/SFH system, and illustrates the delay performance with different modulation types considered here for different frequency hops and as a function of the throughput. As well as we plot the delay as a function of the offered traffic.

\section{Numerical Results}

The system throughput $\mathrm{S}$ and the system delay $\mathrm{D}$ (in time slots) are computed against the offered traffic for the hybrid SFH/DS system. The throughput and delay are computed for DS and SFH by adapting the hybrid SFH/DS system analytical expressions. In the case of pure DS all users use the same carrier frequency; and hence, the number of hopping frequencies $q=1$, the number of active interferers is then equal to the number of active transmitters and the probability of having $n_{i}$ active interferes $P\left(n_{i}\right)=1$. In the case of pure $S F H$, the DS part has left out of the model by taking $\mathrm{N}=1$. the number of resolvable paths, $\mathrm{L}=1$ because there is no inherent spread spectrum anymore.

Figure 3 shows the plot of equations (12), (14), and (16). It gives the bit error rate in $\mathrm{dB}$ as a function of SNR ranged from 0 t0 $14 \mathrm{~dB}$, which illustrate the BER performance of the hybrid SFH/DS SSMA with different considered modulations. Assuming $K=10$ active users, number of hopping frequencies $\mathrm{q}=32$ which is equal to the $\mathrm{DS}$ spread factor $\mathrm{N}=32$, the number of bits per frequency hop $\mathrm{N}_{b}=50$. It is seen from the figure that for the SNR less than $10 \mathrm{~dB}$ the DPSK outperforms the M-FSK where $M=4$, which is better than the BFSK. For higher SNR greater than $10 \mathrm{~dB}$ the M-FSK is the best modulation type then followed by the DPSK while the BFSK still the lowest performance.

Figure 4 gives an indication about the hybrid SFH/DS SSMA system capacity, when plotting the probability of errors as a function of the number of active users ranging from 1 to 150 active users, fixing the SNR at $10 \mathrm{~dB}$, while the number of hopping frequencies $\mathrm{q}=32$, which is equal to the DS spreading factor $\mathrm{N}=32$, and the modulation order $\mathrm{M}=4$ for the $\mathrm{M}$-ary FSK. It is shown from the plot that the M-ary FSK outperform the two other types of modulations from the point of view of the number of users, and then followed by the DPSK, while the BFSK still achieves the minimum number of users.

In Fig. 5 the system throughput is plotted as a function of the total traffic $G$ given that the number of hopping frequencies hop $\mathrm{q}=32$, the DS spread factor $\mathrm{N}=32$, the mean transmission delay in slots assuming 10 active users, number of bits per frequency hop $\mathrm{N}_{\mathrm{b}}=50$, at $\mathrm{SNR}=7 \mathrm{~dB}$. It is seen in the figure that the throughput of the system employing DPSK is better than that of the MFSK which is also better than the BFSK, this is due to the fact that the BER of the DPSK at SNR $=8 \mathrm{~dB}$ is the best followed by the MFSK and BFSK, so as the BER is better the number of packets received correctly increases so the system throughput of the DPSK is the best at this value. Also it is noted from the figure that the throughput increases with the increase of the offered traffic, until certain point at which collision between competing packets occurs then the throughput is decreasing. In Fig.6, the system delay (in 
time slots) is plotted as a function of the offered traffic with the same parameter of Fig.5. it is noted that the delay increase with the increase of the offered traffic, also the delay of the hybrid system employing BSFK is greater than that of the MFSK which is also greater than that of the system employing DPSK.

We repeat the plot of $\mathrm{S}$ against $\mathrm{G}$ in Fig.7 and $\mathrm{D}$ versus $\mathrm{G}$ in Fig.8with the same parameters as in Fig.5 and Fig. 6 but we increase the SNR to $10 \mathrm{~dB}$. It is noted that the BER is very small at this value then the probability of correct reception is $=0.805,0.995$, and 0.996 for the BFSK, MFSK and DPSK respectively. Thus the throughput of the system employing MFSK and DPSK is approximately the same and is lightly better than that of the system employing BFSK. Also there are little differences between the delays of the three modulation types.

In Fig. 9 the plot of $S$ versus $G$ is performed for pure DS system when we adapt the number of frequency hops to $q=1$. It is noted that with the same parameters as before and at $S N R=7 \mathrm{~dB}$ the throughput is more degraded when the DS employs the BFSK. The best throughput is obtained when the DS employs the M-FSK, followed by the DPSK. In Fig.10 the delay of the DS is larger when using the BFSK followed by the DSPK and the smallest delay is achieved when using the M-FSK.

In Fig.11 the plot of $S$ versus $G$ is performed for pure DS system when we adapt the number of frequency hops to $\mathrm{q}=1$. It is noted that with the same parameters as before and at $\mathrm{SNR}=10 \mathrm{~dB}$ the throughput is better than that at $\mathrm{SNR}=7 \mathrm{~dB}$. This is due to the fact that the better the BER performance the better the throughput performance. But still the minimum throughput is obtained when the DS employ the BFSK. The best throughput is obtained when the DS employ the M-FSK, followed by the DPSK. In Fig.12 the delay of the DS is larger when using the BFSK followed by the DSPK and the smallest delay is achieved when using the M-FSK.

In Fig.13 the plot of $S$ versus $G$ is performed for pure SFH system when we adapt the DS spread factor $\mathrm{N}=1$, and at $\mathrm{SNR}=7 \mathrm{~dB}$. It is noted that with the same parameters as before the throughput is degraded when the SFH employs the BFSK but it is better than the pure DS with BFSK. The best throughput is obtained when the SFH employs the M-FSK, followed by the DPSK but it is less than the maximum throughput of the DS system with MFSK or DPSK. In Fig. 14 the delay of the DS is larger when using the BFSK but less than that of the DS with BFSK,, followed by the DSPK and the smallest delay is achieved when using the M-FSK.

In Fig.15 the plot of $S$ versus $G$ is performed for pure SFH system when we adapt the DS spread factor $\mathrm{N}=1$, and at $\mathrm{SNR}=10 \mathrm{~dB}$. The overall throughput is increased due to the increase of the SNR which leads to the enhancement of the BER. The best throughput is obtained when the SFH employs the M-FSK, followed by the DPSK but it is less than the maximum throughput of the DS system with MFSK or DPSK. In Fig.16 the delay of the SFH is larger when using the BFSK, followed by the DSPK and the smallest delay is achieved when using the M-FSK.

\section{Conclusion}

Throughout this paper we derived closed form expressions for the BER and throughput for the hybrid SFH/DS system employing the BFSK, M-ary FSK and DPSK modulations. The system throughput $\mathrm{S}$ (in packet/slots) and the system delay D (in time slots) are computed against the offered traffic (packets/slots) for the hybrid SFH/DS system, also the throughput 
and delay are computed for DS and SFH by adapting the hybrid SFH/DS system analytical expressions with respect to the number of frequency hops and the DS spreading factor.

It is found that the hybrid SFH/DS SSMA system outperform pure DS and pure SFH in different cases. Also it is concluded that the different multiple access techniques when using the BFSK modulation type will provide the lowest performance with respect to the BER, the throughput and system delay. The BER with system employing DPSK is the best performance for SNR < $10 \mathrm{~dB}$ followed by the MFSK, and then BFSK, while the BER for SNR $\geq 10 \mathrm{~dB}$ the system employing the MFSK is the best performance followed by the DPSK, still the BFSK has the lowest performance. Also it is found that the better the BER the better the system throughput and the lower the system delay. Also it is noted from all the previous figures that the throughput increases with the increase of the offered traffic, until a certain point at which collision between competing packets occurs then the throughput is decreasing

\section{References}

[1] John G. Proakis ," Digital Communications", McGraw -Hill, 2006.

[2] Kah C. The, and Kwok H. Li, "Error Probabilities of an FFH/BFSK self normalizing receiver in a Rician fading channel with multitone jamming", IEEE Trans. On Comm., Vol 48, No. 2 February 2000.

[3] E. A Geraniotis and M. B. Pursley,"Error probabilities for slow frequency hopped spread spectrum multiple access communications over fading channels," IEEE Trans. Comm., Vol. COM-30, PP.996-1009, 1982

[4] Heung Gyoon Ryn " Performance of DS/SFH SSMA system with overlapping BFSK in the presence of both MTJ and MAI", IEEE 2003

[5] Zhenhui Tan," Bounds on Performance of RS Coded Hybrid DS-SFH Spread Spectrum Multiple Access for Indoor Wireless Communications", International Conference on Communication Technology ICCT'93.

[6] R. Parsad, Howard S Misser and Antonius Daryanto, "Throughput and delay analysis of a slotted DS/SS CDMA system in a shadowed Rician channel", International conference on Communication Technology ICCT'93, Sept. 1993.

[7] Xiaogang Yu, Hua Wang, Hui Xu, Jingming Kuang " Frequency Occupation and Throughput Analysis of Hybrid spread spectrum DS/FH Network" ,Advanced Information Network and Applications, 2003 ANINA 2003 17th PP. 547- 550.

[8] R. Parsad, "CDMA for wireless personal communications", Artech house INC,1996 


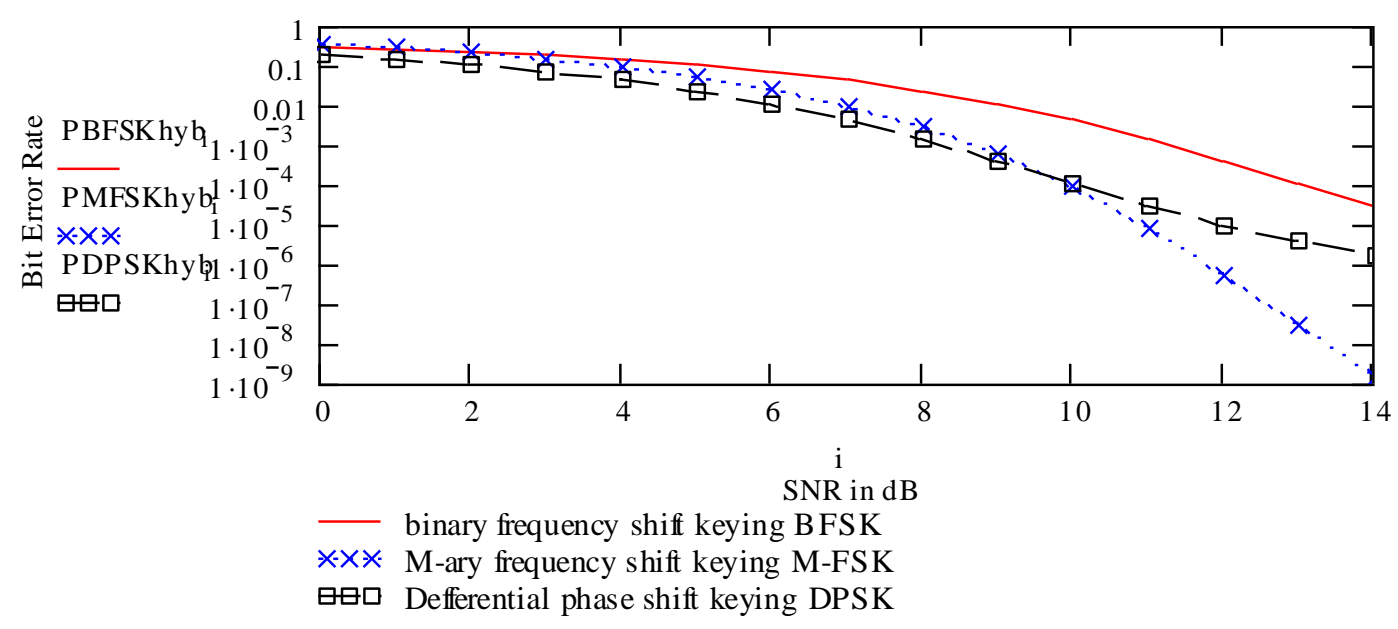

Fig. 3 Hybrid SFH/DS BER performance using BFSK, DPSK and M-FSK with $M=4$, over AWGN channel.

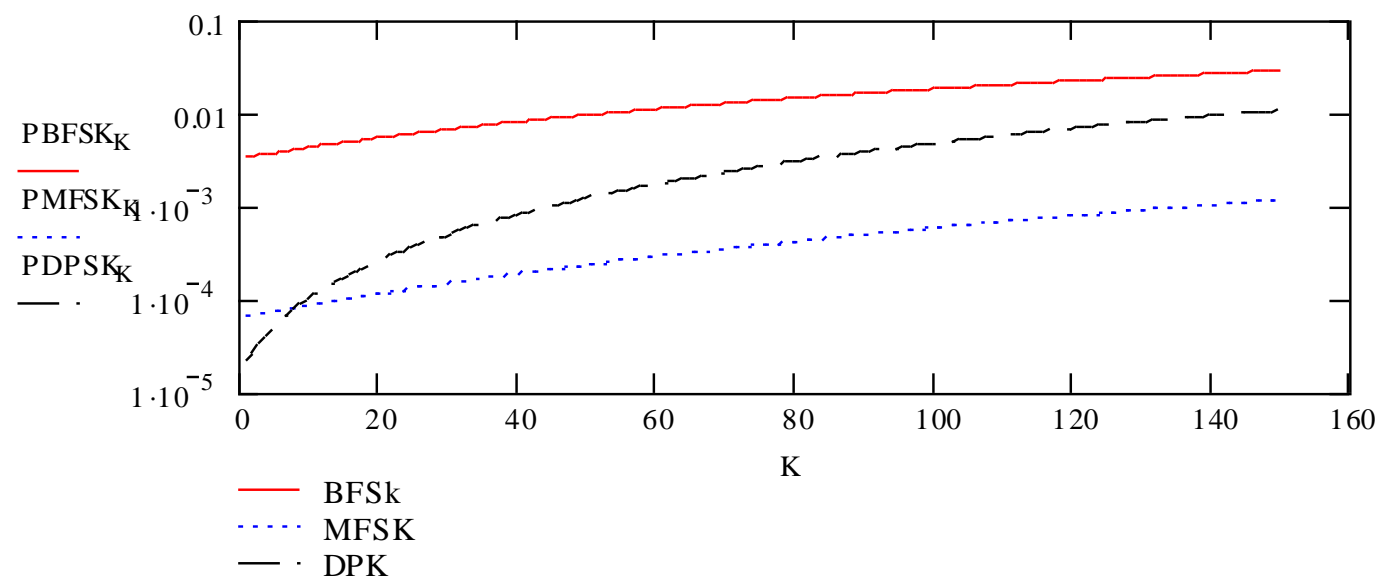

Fig. 4 The probability of error as a function of the number of user for the hybrid SFH/DS SSMA with BFSK, DPSK and MFSK, modulations, with $M=4$.

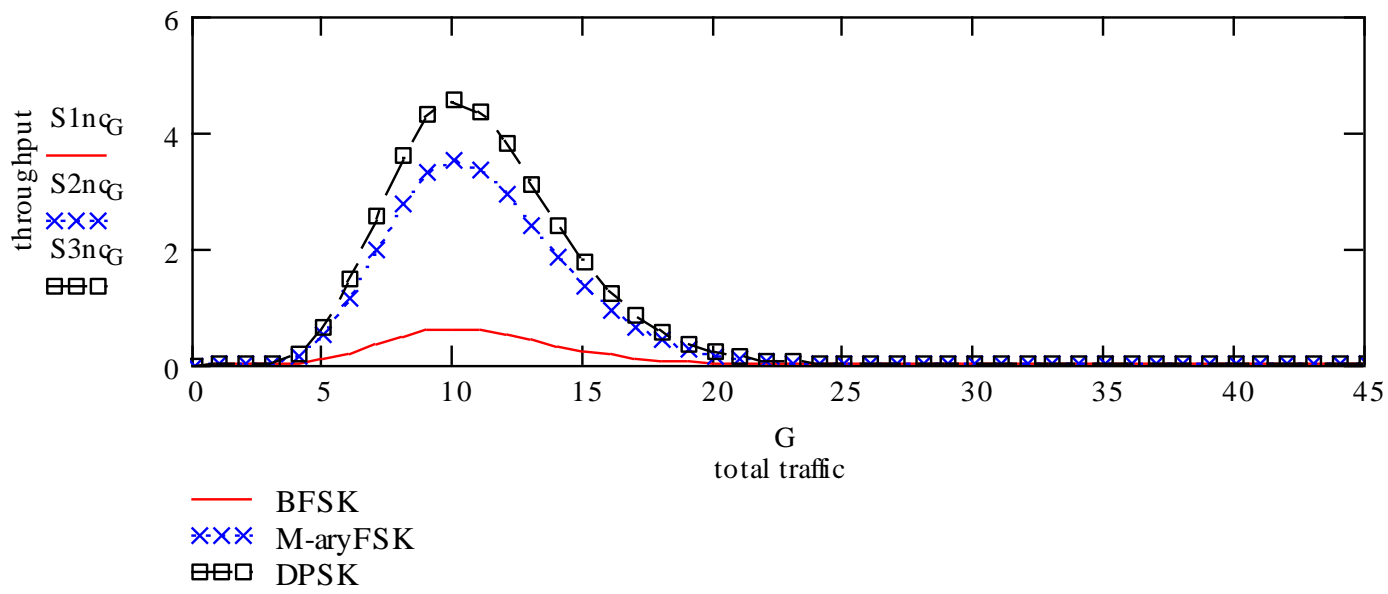

Fig. 5 Throughput against total traffic for hybrid SFH/DS using BFSK, DPSK and $M-F S K$, and $M=4$ at $\mathrm{SNR}=7 \mathrm{~dB}$. 


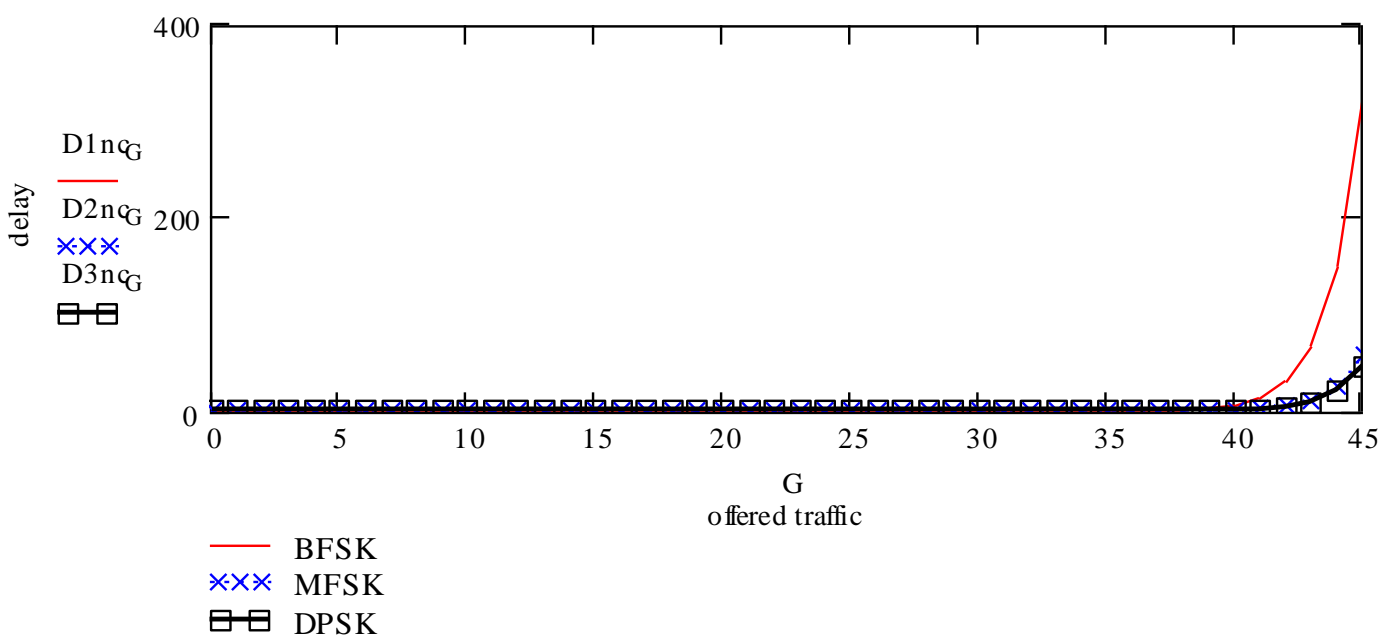

Fig. 6 System delay versus offered traffic for the hybrid SFH/DS for BFSK, MFSK, and DPSK at $\mathrm{SNR}=7 \mathrm{~dB}$.

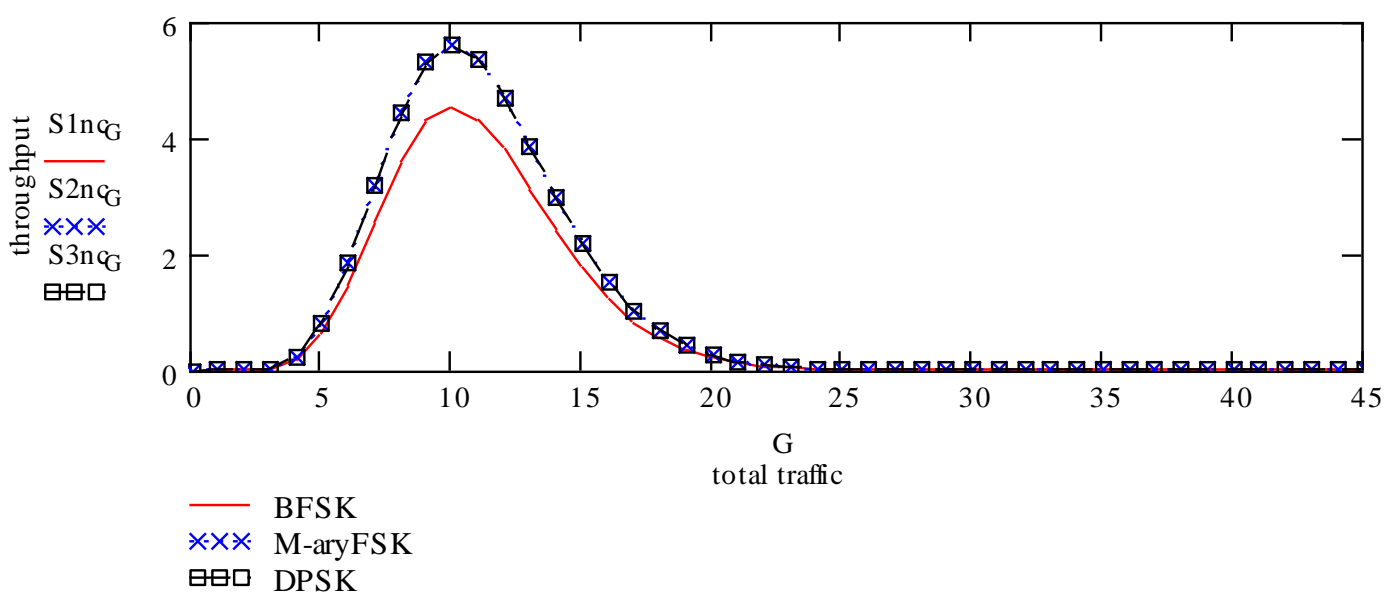

Fig. 7 Throughput performance against total traffic for the hybrid SFH/DS for BFSK, MFSK, and DPSK at SNR $\geq 10 \mathrm{~dB}$.

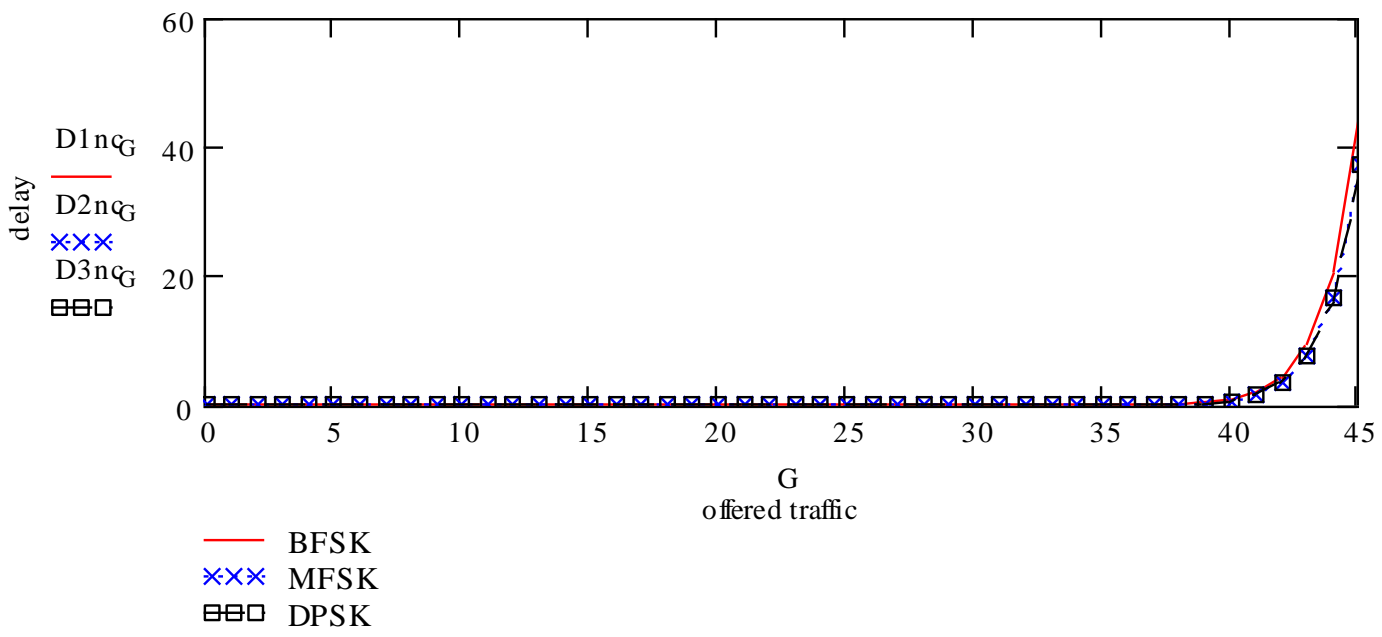

Fig. 8 System delay versus offered traffic for the hybrid SFH/DS for BFSK, MFSK, and DPSK at SNR $\geq 10 \mathrm{~dB}$ 


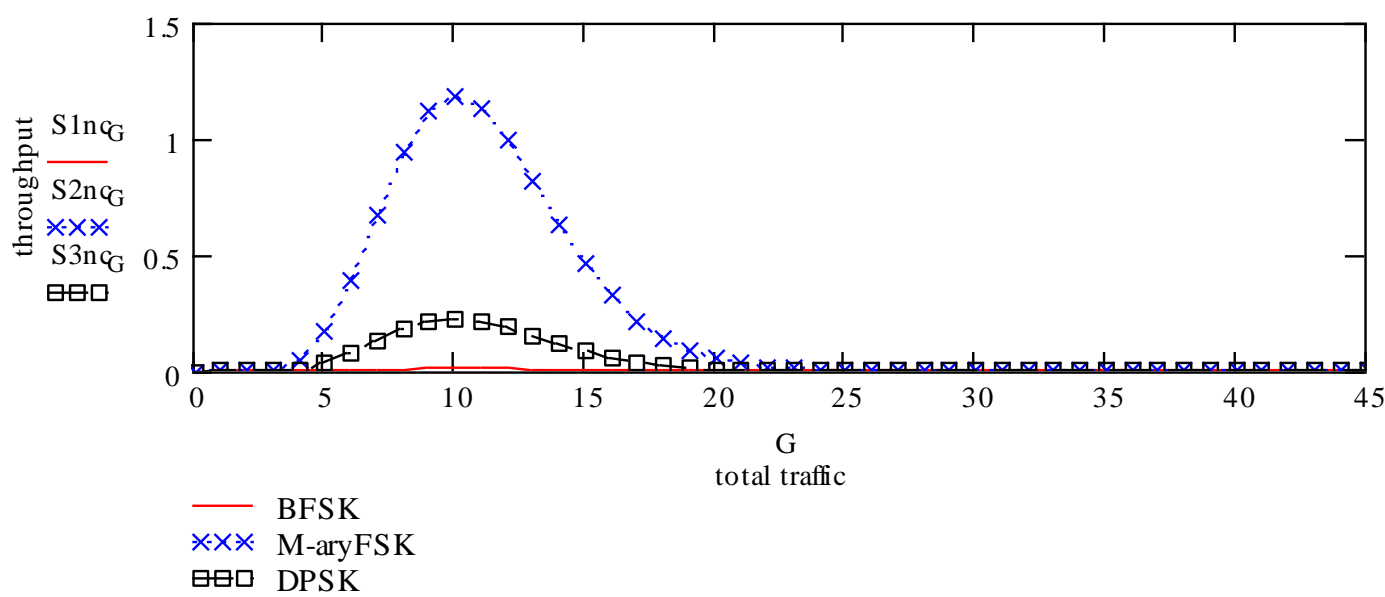

Fig. 9 Throughput performance against total traffic for the pure DS with BFSK, MFSK, and DPSK at $\mathrm{SNR}=7 \mathrm{~dB}$.

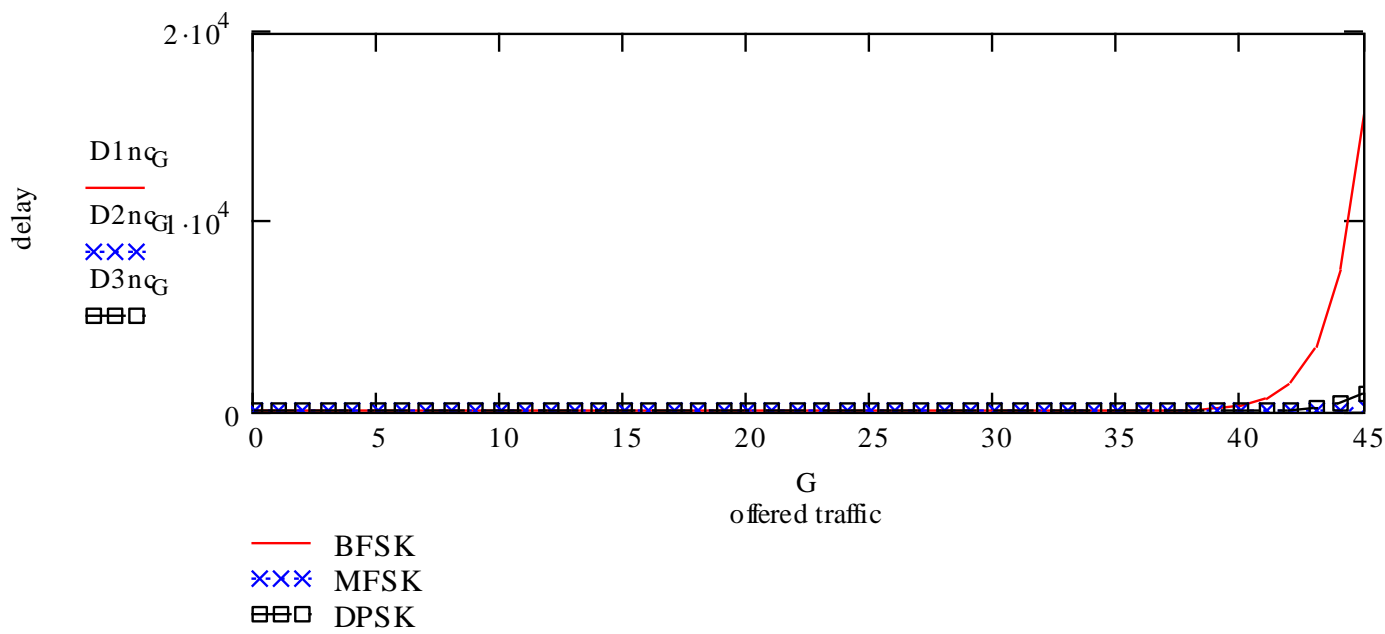

Fig. 10 System delay versus offered traffic for the pure DS with BFSK, MFSK, and DPSK at SNR = $7 \mathrm{~dB}$

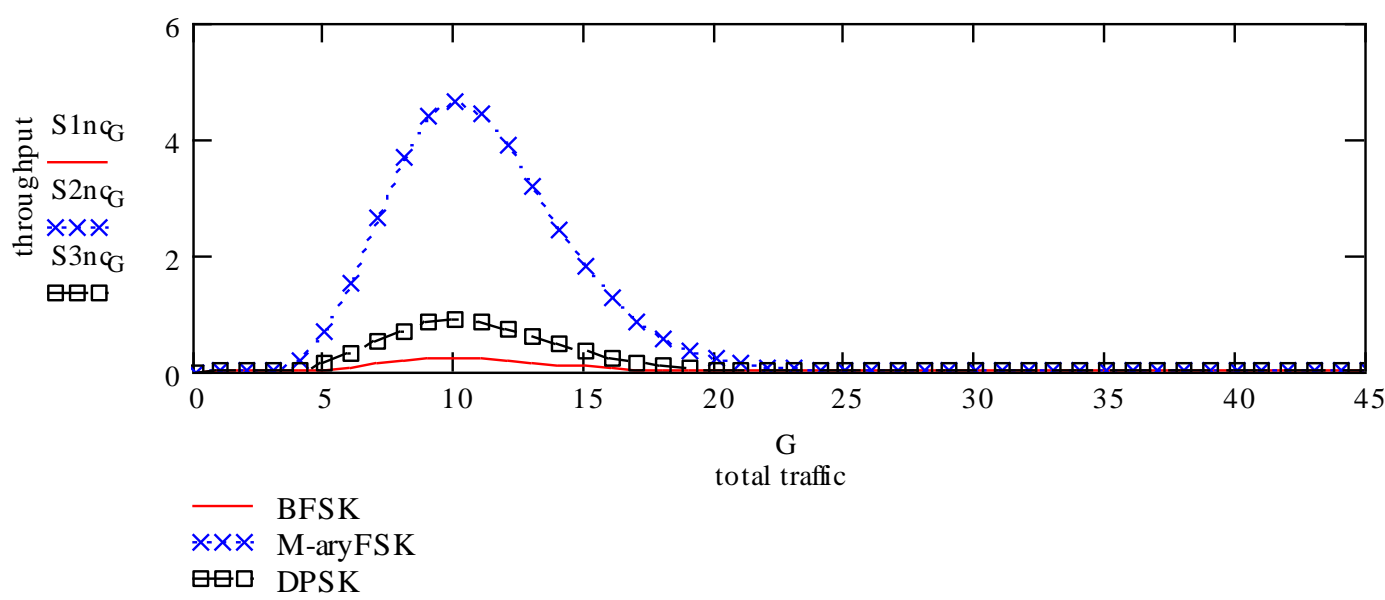

Fig. 11 Throughput performance against total traffic for the pure DS with BFSK, MFSK, and DPSK at SNR $\geq 10 \mathrm{~dB}$ 


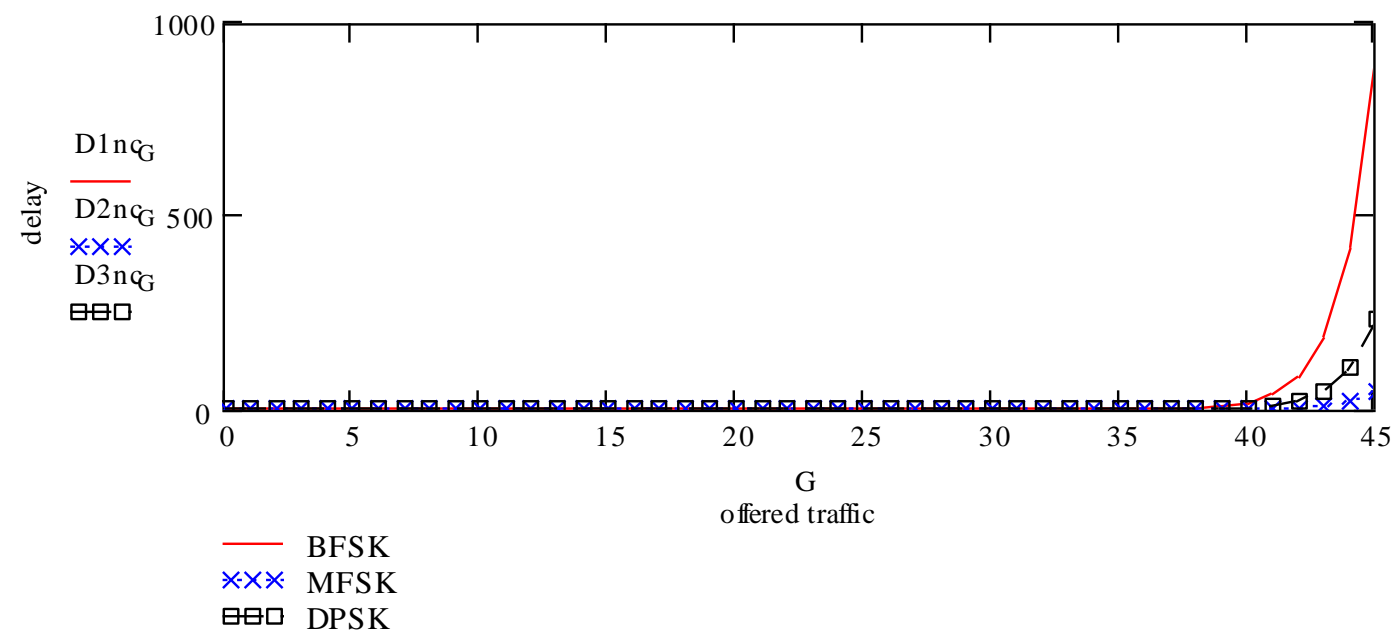

Fig. 12 System delay versus offered traffic for the pure DS with BFSK, MFSK, and DPSK at SNR $\geq 10 \mathrm{~dB}$.

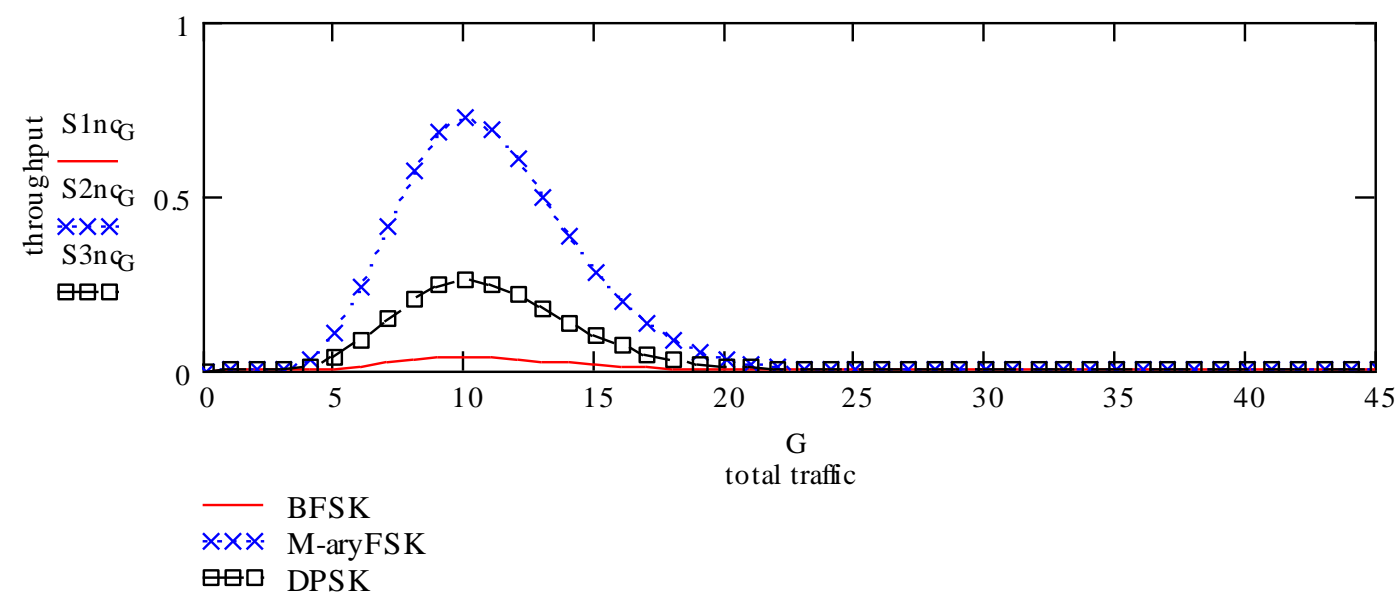

Fig. 13 Throughput performance against total traffic for the pure SFH with BFSK, MFSK, and DPSK at SNR=7 dB.

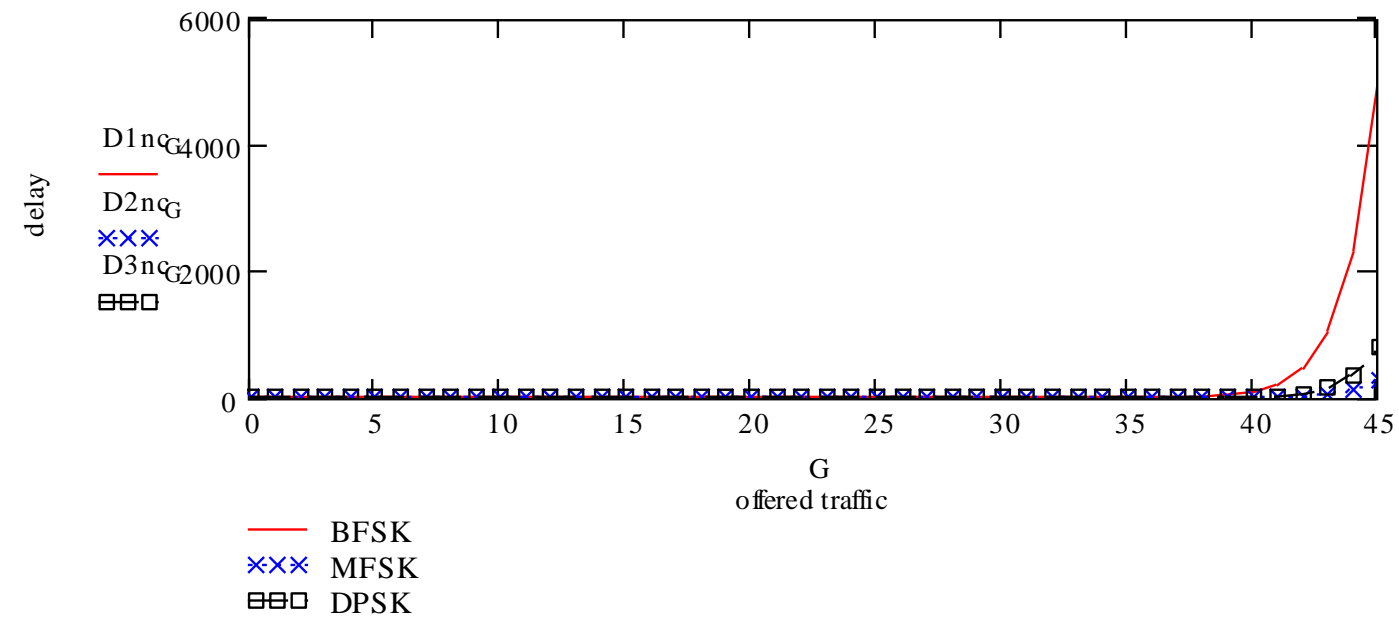

Fig. 14 System delay versus offered traffic for the pure SFH with BFSK, MFSK, and DPSK at SNR=7 dB. 


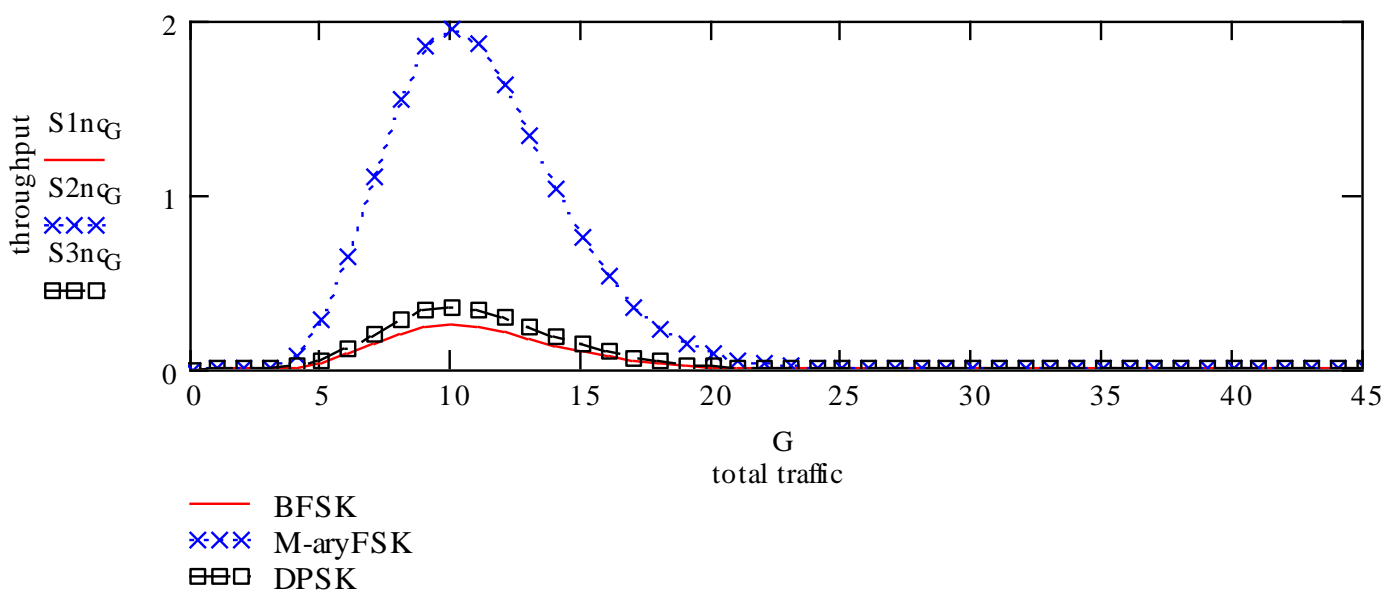

Fig. 15 Throughput performance against total traffic for the pure SFH with BFSK, MFSK, and DPSK at SNR $\geq 10 \mathrm{~dB}$.

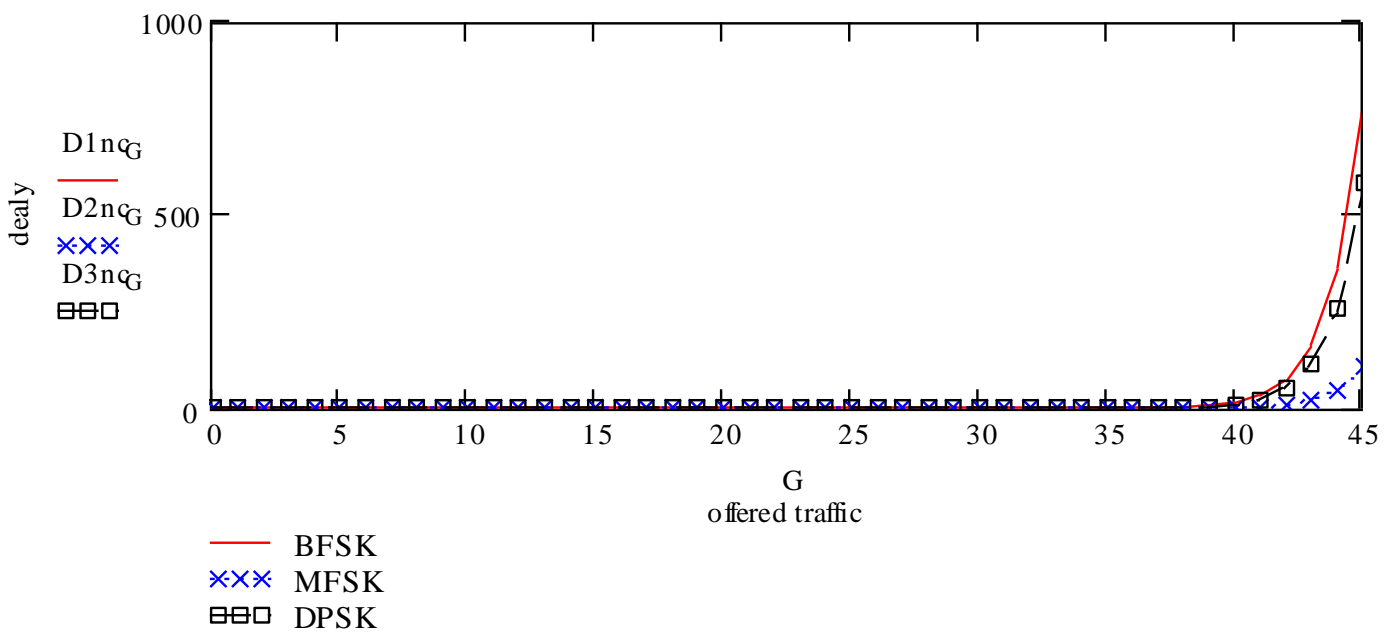

Fig. 16 System delay versus offered traffic for the pure SFH with BFSK, MFSK, and DPSK at SNR $\geq 10 \mathrm{~dB}$. 